\title{
THE NEED OF GROUND-CORRELATED HIGH-RESOLUTION SPACE OBSERVATIONS IN THE VISIBLE*
}

\author{
K.O. KIEPENHEUER \\ (Fraunhofer Institute, Freiburg i. Br., Germany)
}

Solar Research during the last decade has definitely shown that almost all primary processes which build up the chromosphere and the inner corona, as well as the observable basic events leading to the optical manifestation of solar activity occur practically all in the subtelescopic range, as far as ground-based observation is concerned. I. think here mainly of the great variety of phenomena occurring in the intergranular space (width $<0$ ".3) and intersupergranular space (width $<1^{\prime \prime}$ ). Because of this situation we have only very few reliable information about the structural transition between photosphere and chromosphere, how the granulation goes over into the complex chromospheric structures as bright and dark mottles, spicules, threads, loops, or fibrils. In this region theory can still get along without being disturbed too much by observation. The situation is somewhat like looking into a human face with a resolving power of 1 or $2 \mathrm{~cm}$ !

For the magnetic field - nobody doubts its crucial importance - the situation is still much worse. Every scientist who has not been brought up - like most of us here - with the implied agreement to get along with our atmosphere without complaint, would first try to create the conditions which would enable him to resolve $0^{\prime \prime} .1$ or $0^{\prime \prime} .2$ and then go on with solar research. For the ground-based solar observer $1^{\prime \prime}$ or 0 ".5 are already something extraordinary (at least for monochromatic images or high-resolution spectrograms). There is no hope for him to reach $0^{\prime \prime} .2$ or $0^{\prime \prime} .1$. And it is astonishing to see with how much patience this sad fact is being accepted.

One way out is balloon astronomy, another one observing in space outside the atmosphere. It must be made quite clear here that the gain of angular resolution by observing from the stratosphere or from space is by no means less important than the extension of the spectrum in the EUV- or X-region. Both will bring us an unpredictable wealth of information.

The balloon technique - first brought to success by Schwarzschild and his group has the advantage that the instrument can be flown several times; instrumental improvements can be brought in this way. However, balloons with such heavy payloads

* Mitteilungen aus dem Fraunhofer Institut Nr. 77.

Perek (ed.), Highlights of Astronomy, 527-529. (C) I.A.U. 
cannot be launched at short notice. Waiting times of the order of weeks might arrive because of weather situations at the launching site or along the balloon's trajectory. The observing time from a balloon can be 8 hours. Angular resolutions better than 0.3 or $0 " 2$ in the presence of sunlight might meet with serious difficulties because of the remaining air around the instrument.

In contrast to the balloon projects, the manned ATM project - and only about this I will speak here - will offer an observing period of $\sim 50$ days. This implies a significant probability that

(1) special events like larger flares, formation of sunspots etc., will occur during the mission;

(2) the astronauts have enough time to get adapted to their TV monitor and to a fruitful communication with ground-based astronomers; and

(3) the necessary adjustments or changes of program or necessary repairs can be made during flight.

Without ignoring the great value of the existing high-resolution projects on the ground or in the stratosphere - they will always be indispensable and form the intermediate steps to obtaining better optical high-resolution results in space - I would like to plead here strongly to use even the ATM facilities for getting the highest possible angular resolution in the visible part of the spectrum. By combining a prime mirror of $100 \mathrm{~cm}$ aperture with a high-resolution spectrograph and a Lyot filter (for better locating the position of the spectrographic slit on the Sun) an angular resolution of 0.1 can be reached, which will be equivalent to an increase of flux of information (per unit of the Sun's surface) by a factor 100 as compared to the best conditions on ground. The guiding of such an instrument will certainly not be an easy problem. However, the experiences which we have collected with our balloon-borne spectrostratoscope, which is locked in solar granules, look quite promising.

A film size of $60 \times 60 \mathrm{~mm}$ will correspond for this high resolution to a field of $1^{\prime} \times 1^{\prime}$ on the Sun. In other words, this instrument will be a kind of 'space microscope' in the hands of the cooperating ground-based astronomer, handled through his space fellow astronaut.

The amount of pairs of spectrograms and pictures to be obtained during a 50-days mission could be of the order of 400000 ( $24000 \mathrm{~m}$ of film) and could be supplied after return to the ground to a number of observatories for examination and evaluation.

The possibility of investigating flares with such a 'space microscope' needs special mentioning. Flare theories have become an exciting battle field of advanced theoreticians, while unfortunately, flare observation is still in its very infancy. Most of the observers are convinced that the initial and basic process of a flare, as well as its close environment has not yet been observed with adequate angular resolution; we are at least off by one order of magnitude. The probability to succeed with a balloon-borne instrument during an 8 hours' flight is very small. The coincidence of a flare with excellent seeing on the ground is still more improbable. During the 50-day ATM ob- 
serving period it should be possible (even with the small field available) by continuous contact between the operating astronaut and a competent group on the ground, to follow a flare from its very beginning (structure, spectrum and magnetic field) down to dimensions of $\$ 100 \mathrm{~km}$. Although the magnetic-field pattern probably cannot be resolved down to $0^{\prime \prime} 1$, the fine structure (as seen in certain lines or in integrated light) together with somewhat less resolved magnetograms will give us an idea of the magnetic fine structure.

There is no need to mention that apart from getting the highest possible angular resolution (which again for many reasons should be first practised in the visible) additional information in the EUV will be of greatest importance, especially if correlated to better than $1^{\prime \prime}$ to the visible features.

I think that the community of solar physicists all over the world would appreciate enormously, if NASA could make available such a 'space microscope' to supplement ground and balloon efforts. 